\section{Fünf Jahre IATAFI}

\author{
von Reinhard Coenen, ITAS
}

Die International Association of Technology Assessment and Forecasting Institutions (IATAFI) wurde 1993 unter der Schirmherrschaft der Vereinten Nationen in Bergen, Norwegen, gegründet und schaut damit im November 1998 auf ihrer dritten Bi-Annual Conference in New Delhi, Indien, auf die ersten fünf Jahre ihres Bestehens zurück. Die Konferenz widmet sich dem Thema: "Equity, Energy and Environment: Challenges for Technology Assessment and Forecasting".

Ein zentrales Ziel der IATAFI ist es, weltweit die Zusammenarbeit und den Erfahrungsaustausch zwischen Technology Assessment and Forecasting-Einrichtungen zu fördern und dabei insbesondere entsprechende Einrichtungen in den Ländern der Dritten Welt zu fördern. Die Ziele im einzelnen sind:

- Link the countries which lack TA (mostly developing countries) to TA/TF capabilities in developed countries.

- Promote technology assessment (including environmental assessment) and forecasting in the decision-making process.

- Establish regular communication links and share information among all Association members (including electronic mail).

- Collaborate in technology assessment by creating an opportunity for members to participate in, or contribute to education and analysis being carried out by other members.

- Exchange personnel for training in TA in order to strengthen or build skills and expertise.

- Co-ordinate collateral activities, procedures and methodologies.

- Co-operate with international activities being carried out under the global change agenda

(IATAFI 1994 - 1998, November 1998).

IATAFI verfügt gegenwärtig über 37 Mitgliedsorganisationen, die sich aus 27 Einrichtungen aus Industrieländern und 10 Einrichtungen aus
Entwicklungs- oder Schwellenländern zusammensetzen. ITAS ist seit Gründung Mitglied. Hervorzuheben ist, daß es gelungen ist, wichtige und zentrale Einrichtungen in Entwicklungsländern als sehr aktive Mitglieder zu gewinnen. So wird die diesjährige Konferenz vom indischen Technological Information, Forecasting and Assessment Council (TIFAC) veranstaltet, der letztjährige IATAFI Workshop on "Zero Emission and Technology Assessment in a Global World" wurde vom Centre Tecnologia Mineral (CETEM) in Brasilien organisiert. IATAFI finanziert sich einerseits aus Mitgliedsbeiträgen, andererseits wurde IATAFI bisher finanziell und technisch durch das internationale Ölunternehmen Statoil mit Stammsitz in Bergen, Norwegen, unterstützt. Darüber hinaus bietet der Hordaland County Council Unterstützung, in dem er das IATAFI-Sekretariat beherbergt. Außerdem wurde die Durchführung von Konferenzen und Workshops des IATAFI von verschiedenen Organisationen finanziell unterstützt, so z.B. durch verschiedene norwegische Regierungsstellen, die EU-Kommission, das NATO-Science Committee sowie durch die für die IATAFI-Veranstaltungen gastgebenden Einrichtungen.

Zieht man eine Bilanz der Arbeit von IATAFI, so fällt diese geteilt aus, was nicht am fehlenden Engagement der Mitgliedseinrichtungen des Executive Board liegt, sondern an den finanziellen Restriktionen, unter denen IATAFI bisher operieren mußte. Durch die von IATAFI veranstalteten Workshops und Konferenzen wurde zweifellos der Erfahrungsaustausch zwischen den TA-Einrichtungen aus verschiedenen Regionen der Welt gefördert. IATAFI nutzt auch intensiv neue Kommunikationsmedien wie das INTERNET und Telefon-Konferenzen, um die Kontakte zwischen den Mitgliedsorganisationen zu pflegen und zu intensivieren. IATAFI publiziert viermal jährlich den Newsletter FUTURUM, der sich auf Beiträge der Mitgliedseinrichtungen stützt, und bietet auf seiner World-Wide-Web Seite TA-relevante Informationen an. Vereinzelt konnten auch Kooperationen zwischen Forschungseinrichtungen aus Industrie- und Entwicklungsländern angeregt und der Austausch von Fachleuten zwischen Mitgliedsorganisationen (z.B. zwischen Einrichtungen in den USA und China) unterstützt werden. IATAFI hat sich im Rahmen von klei- 
neren Workshops auch darum bemüht, im Sitzland Norwegen die Diskussion um die Institutionalisierung von TA anzustoßen und weiterzubringen. Die Durchführung gemeinsamer Projekte sowie von größeren Trainings- und Wissenschaftleraustausch-Programmen scheiterte jedoch bisher an den finanziellen Restriktionen. Sofern sich an der finanziellen Situation nichts Entscheidendes ändert, wird sich IATAFI wohl auch in Zukunft auf die Veranstaltung von Workshops und Tagungen mit Unterstützung von Sponsoren und die Nutzung des Internets zum Informationsaustausch zwischen den Mitgliedseinrichtungen und zur Verbreitung von TA-relevanten Informationen beschränken müssen. Die anläßlich der Konferenz in New Delhi stattfindenden Sitzungen der Mitgliederversammlung und des Executive Board werden sich intensiv mit den zukünftigen Weichenstellungen für die Arbeit von IATAFI beschäftigen müssen.

\section{Kontakt}

Jan Anderson

President of IATAFI

The International Association for Technology Assessment and Forecasting Institutions

c/o The European Office

Hordaland County Council

N-5025 Bergen

Tel.: 55239534

Fax: 5555239379

E-mail: iatafi@iatafi.no

Web: www.iatafi.no

Reinhard Coenen

Executive Secretary IATAFI

Forschungszentrum Karlsruhe

Institut für Technikfolgenabschätzung

und Systemanalyse (ITAS)

Postfach 36 40, D-76021 Karlsruhe

Tel.: ++ 49 (0) 7247/82-2509

Fax: ++ 49 (0) 7247/82-4806

E-mail: Coenen@itas.fzk.de

\section{TA-CD-ROM Bestellschein}

Fax-Nr.: +49(0)7247 / 824806

Ich bestelle .......... Exemplar(e) der TA-CD-ROM zum Einzelpreis von 65 DM (inkl. MwSt.).

Name:

Einrichtung:

Adresse:

Datum: Unterschrift: 